\title{
Le paysage et l'expérience du numineux dans les récits autobiographiques de George Sand
}

\section{Carlota Vicens-Pujol}

\section{(2) OpenEdition}

1 Journals

\section{Édition électronique}

URL : http://journals.openedition.org/studifrancesi/10772

DOI : 10.4000/studifrancesi. 10772

ISSN : 2421-5856

Éditeur

Rosenberg \& Sellier

\section{Édition imprimée}

Date de publication : 1 avril 2018

Pagination : 41-50

ISSN : 0039-2944

\section{Référence électronique}

Carlota Vicens-Pujol, « Le paysage et l'expérience du numineux dans les récits autobiographiques de George Sand », Studi Francesi [En ligne], 184 (LXII | I) | 2018, mis en ligne le 01 avril 2019, consulté le 06 janvier 2021. URL : http://journals.openedition.org/studifrancesi/10772 ; DOI : https://doi.org/ 10.4000/studifrancesi. 10772

\section{(c) (i) $\odot$}

Studi Francesi è distribuita con Licenza Creative Commons Attribuzione - Non commerciale - Non opere derivate 4.0 Internazionale. 


\title{
Le paysage et l'expérience du numineux dans les récits autobiographiques de George Sand
}

\begin{abstract}
In 1917 Rudolf Otto proposed the word "numinous" to denote the irrational side of religious experience, experience that is an apprehension of a mysterium tremendum et fascinans. In the same way Edmund Burke or Immanuel Kant, who theorised the aesthetics of the sublime, spoke of this kind of delicious horror that man feels when faced with that which surpasses him and makes him aware of his smallness before enormity, defined by Otto as «that which is not normal, that which is disturbing». The sublime sombre of romantic landscapes and the numinous appear to be very closely-linked concepts. Moreover, George Sand always displayed religious and spiritual concerns which, whilst they may have evolved over the years, are nevertheless still successors to the theories of Pierre Leroux or Robert de Lamennais. However, one can distinguish in these texts a need for the Absolute, an impetus towards God revealed in the descriptions of landscapes. On the following pages our aim was, moving away from the writer's novelistic production, to ponder over the Sandian landscapes as described in her travel diaries and in other autobiographical texts. We will see how the way George Sand experienced nature and the grand brings her nearer to this absolute presence which enables us to speak of experience of the numinous. A strong presence of Christian symbolism confirms the presence of a bomo religiosus behind the writer. The landscapes described in this way show themselves to be sites steeped in numinous power where the narrator encounters the Wholly Other.
\end{abstract}

«Que sais-je? On ne voit pas bien, on ne peut pas regarder assez. Il y a trop d'étonnement»

«Tout cela m'a paru horrible et délicieux en même temps. J'avais peur, une peur inouïe et sans cause, une peur de vertige et qui n'était pas sans charme»

Histoire de ma vie, George Sand

\section{Introduction}

En 1917 le théologien allemand Rudolf Otto proposait dans Le Sacré. L'élément non rationnel dans l'idée du divin et sa relation avec le rationnel le mot «numineux» pour désigner l'aspect irrationnel de l'expérience religieuse, de toute émotion religieuse fortement ressentie. Cette rencontre avec le «Tout Autre», avec le «tout à fait hétérogène» est définie comme mysterium tremendum et fascinans: la terreur qu'il inspire, et qui ne peut naître de rien de terrestre, de connu, est accompagnée d'un désir d'Absolu éprouvé avec une force telle qu'il est impossible de s'y soustraire.

Dans sa définition du sacré Jean-Jacques Wunenburger résume très bien cette notion somme toute abstraite sur laquelle on reviendra. Rudolf Otto, écrit-il,

propose d'identifier le sacré par des sentiments (le numineux) ambivalents d'attirance et de répulsion (majestas et tremendum) qui sont accompagnés de représentations intellectuelles [...]. 
R. Otto rattache le sacré à une structure émotionnelle a priori, le numinosum, qui se rapporte à l'impression qu'a la conscience d'être conditionnée par une force indépendante de sa volonté, le Tout Autre [...]. L'expérience du numineux apparaît ambivalente et bipolaire: d'un côté le numineux est relation à un Mysterium tremendum, sensation d'effroi panique devant une grandeur incommensurable ou une puissance souveraine; d'un autre côté il est appréhension d'un Mysterium fascinans, qui s'exprime par des forces d'attraction vers quelque chose de merveilleux et de solennel. Le prisme émotionnel du numineux s'étend donc depuis la réaction devant l'horrible jusqu'à celle devant le sublime ${ }^{1}$.

De leur côté Edmund Burke ou Emmanuel Kant, ou encore Longin un siècle auparavant dans la traduction de Boileau², ont théorisé l'esthétique du sublime qui s'imposa avec force au XVIII ${ }^{\mathrm{e}}$ siècle: opposé au beau, le sublime se manifeste lorsque l'homme ressent devant le spectacle de la Nature une sorte d'horreur délicieuse. D'où le sublime sombre, «expression esthétique d'une terreur qui naît de la sensation d'une puissance inconnue et d'autant plus effrayante qu'elle est confuse et diffuse» ${ }^{3}$. Il est vrai que depuis le pseudo-Longin l'idée du sublime a bien évolué. Si au XVII ${ }^{\mathrm{e}}$ siècle elle appartient toujours à l'ordre du discours rhétorique, l'auteur du traité donnera au mot une certaine transcendance ${ }^{4}$. Puis, dès 1757 le mot va définitivement s'affranchir des prescriptions de la stylistique. Comme le signale Yvon Le Scanff, Edmund Burke interprète cette notion «dans le sens d'un sensualisme et d'un intensivisme et [va] promouvoir la terreur comme sensation déterminante en comprenant le parodoxon longinien comme stupéfaction douloureuse $[\ldots] \gg^{5}$. Avec Kant le concept entre dans le domaine du spirituel. Les deux formes de sublime dont il parle (le mathématique, à la limite du beau, et le dynamique, terrifiant) témoignent d'après lui du côté spirituel de l'homme, car le sublime «n'est contenu en aucune chose de la nature, mais seulement dans notre esprit» ${ }^{6}$.

Ces théories, notamment celles de Burke, ont été largement développées par Yvon Le Scanf dans Le paysage romantique et l'expérience du sublime, où il montre comment «ces poétiques du paysage accompagnent l'esthétique burkienne de façon magistrale en mettant en œuvre les éléments fondamentaux de ce sublime sombre: l'horreur chaotique, la terreur et la privation» ${ }^{7}$. La force et l'exubérance de la nature, son aspect le plus sauvage, viennent se substituer à l'ordre et la mesure des siècles précédents, à tel point que le locus borridus deviendra le paysage romantique par excellence. Confronté à lui, l'homme ne peut que sentir son néant, sa petitesse, ainsi qu'une sorte d'immensité non nommée qui le dépasse. Or l'indicible, l'ineffable, l'approchent du sacré.

Dans les pages qui suivent nous voulons nous pencher sur l'expérience que par la voix du narrateur George Sand fait du numineux, de la rencontre du Tout-Autre.

(1) J-J. Wunenburgerg, Le sacré, Paris, PUF, «Que sais-je?», 2009, p. 12.

(2) Dans Recherche philosophique sur l'origine de nos idées du sublime et du beau (1757), Le beau et le sublime (1764) et Traité du sublime (1674) respectivement. Les dates correspondent ici aux premières éditions. Ce dernier traité est également connu comme le pseudo-Longin, car on ne saurait affirmer que Longin en soit vraiment l'auteur.

(3) Y. LE SCAnfF, Le paysage romantique et l'expérience du sublime, Champ Vallon, Seyssel, 2007, p. 31.

(4) «Car tout ce qui est véritablement sublime a cela de propre, quand on l'écoute, qu'il élève l'âme [...] la remplissant de joie et de je ne sais quel noble orgueil, comme si c'était elle qui eût produit les choses qu'elle vient simplement d'entendre» dans (Euvres de Boileau, Paris, Librairie de Firmin Didot frères, 1844.

(5) Y. LE SCANFF, op. cit., p. 28.

(6) E. Kant, dans Critique de la faculté de juger. Cité par Le SCANFF, op. cit., p. 49.

(7) Le SCANFF, op. cit., p. 31. Pour l'évolution de l'idée de sublime et de l'esthétique du sublime jusqu'au romantisme voir D. Peyrache-Laborgne, La poétique du sublime, de la fin des Lumières au romantisme, Paris, Champion, 1997. 
Et ce à partir des descriptions sandiennes du paysage dans son œuvre autobiographique, notamment ses récits de voyages': Lettres d'un Voyageur (1837), Un Hiver à Majorque (1842) ou Voyage en Auvergne, mais aussi de quelques pages choisies de la Correspondance ou d'Histoire de ma vie et de quelques œuvres mineures comme Sketches and hints. On verra comment l'expérience que George Sand fait de la nature et du grandiose l'approche de cette présence absolue, de ce mysterium tremendum et fascinans qui caractérise l'expérience du numineux. Ceci sans oublier les analogies existantes entre le sentiment du sublime (appartenant à l'ordre de l'esthétique) et celui du numineux (appartenant à l'ordre du religieux ${ }^{10}$. Cherchant à dévoiler l'bomo religiosus caché derrière les différents narrateurs, nous nous arrêterons dans un deuxième temps sur la présence de Dieu et de la symbolique chrétienne dans les descriptions de paysages, puis sur tout ce qui dans le discours sandien relève de l'écriture des mystiques chrétiens, aussi bien de l'école rhénane que de l'espagnole.

Soulignons que nous ne tiendrons pas compte ici de l'idéal religieux de George Sand tel qu'elle essaie de le définir - il devient alors rationnel - dans ses articles et romans (comme Le Meunier d'Angibault ou Lélia), très influencés par les théories de Pierre Leroux et, dans une moindre mesure, de Robert de Lamennais, ceci ayant déjà fait l'objet de plusieurs analyses ${ }^{11}$.

\section{Il y a trop d'étonnement}

Aussi bien dans son Traité d'bistoire des religions que dans Le sacré et le profane ${ }^{12}$, Mircea Eliade soutient que tout objet profane est susceptible de devenir «récipient» du sacré, lieu de manifestation du divin. Tout ce que l'homme a touché, senti, trouvé ou aimé peut devenir hiérophanie ${ }^{13}$, écrit-il, pour ajouter que «tout ce qui est insolite, singulier, nouveau, parfait ou monstrueux devient un récipient pour les forces magico-religieuses» ${ }^{14}$. Il établit toutefois plusieurs sortes de hiérophanies; parmi elles, celles qu'on pourrait grouper sous le nom de «cosmiques», notamment le ciel, la mer et la montagne, vont devenir chez les romantiques le lieu privilégié de manifestation de la sublimité, voire de la divinité.

Rousseau avait bien fixé dès la première moitié du dix-huitième siècle les attributs de ce paysage hiérophanique qu'adopteront par la suite les écrivains roman-

(8) Pour une définition du récit de voyage et sa relation avec l'autobiographie, voir R. LE HuENEN: Qu'est-ce qu'un récit de voyage?, «Littérales» 7, 1990, pp. 11-27. De ce même auteur voir aussi «L'écriture romantique du voyage», dans A. Goldschälager, Y. Martineau et C. Thomson, éd. Règles du genre et inventions du génie, Ontario, Mestengo Press, 1999, pp. 219-230.

(9) Ce texte, né d'un premier voyage en Auvergne réalisé en août 1827, ne fut publié qu'en 1888 dans «Le Figaro». George Lubin l'inclut dans son édition des Euvres autobiographiques, Paris, Gallimard, «La Pléiade», 1971. Dorénavant les textes de G. Sand seront désignés dans le corpus du texte avec l'aide des sigles: Voyage en Auvergne (VA), Lettres d'un voyageur (LV), Un Hiver à Majorque (HM), Histoire de ma vie $(\mathrm{HV})$; Sketches and Hind (SH) et Correspondance (C), suivis du numéro de la page.

(10) R. Oтto, Le Sacré. L'élément non rationnel dans l'idée du divin et sa relation avec le rationnel, Paris, Payot, 1969, pp. 72 à 81.

(11) À ce propos voir, entre autres M. Moret, Le sentiment religieux chez George Sand, Paris, Vigné, 1936; F.-P. Bowman, George Sand, le Christ et le royaume, «Cahiers de l'Association Internationale des études françaises» 28, 1976, pp. 243-262 ou encore, A. CAMENISCH, Une croyante spiritualiste: George Sand, «Vives Lettres» 7, 1999, pp. 30-39.

(12) M. Eliade, Tratado de historia de las religiones (dorénavant THR), México, Ed. Era, 1975 et Le sacré et le profane (dorénavant SP), Paris, Gallimard, 1965.

(13) ElIADE, THR, p. 35.

(14) Ibidem, p. 37. 
tiques. Les propos tenus par l'auteur des Confessions ${ }^{15}$ sont repris un siècle plus tard par George Sand:

[...] si le torrent roule quelquefois limpide et calme, si la tristesse y soulève un instant ses sombres voiles et si un pareil site s'avise de vouloir sourire, je le déclare poncif [...], c'est-à-dire, pleutre, manqué, à côté du beau. Je le déshérite de ma sympathie, je lui retire mon souvenir et je tiens pour épiciers et malappris tous les voyageurs qui s'y rendront par un beau temps. $(\mathrm{LV}, 895)$

L'un et l'autre restent bien dans le domaine du beau et non pas, on le voit, dans celui du sacré ${ }^{16}$. Il est vrai que les voyageurs romantiques, dont George Sand, trouveront dans la description des paysages un lieu d'épanouissement du moi, de quête, de saisissement, une expression parfois un peu gauche de leurs aspirations et de leur spiritualité. Chateaubriand, quant à lui, avait bien remarqué qu'il était inutile d'aller chercher l'infini dans la nature, car celui-ci se trouverait (ou ne se trouverait pas) au plus profond de l'homme: le silence et l'immobilité, la rencontre avec soi, l'y amènent. C'est bien ce silence intérieur, source d'infini, beaucoup plus que les «objets extérieurs», que George Sand va peindre dans ses récits. Mais cet infini tant recherché, cet inconditionné, «ne peut être objet d'enseignement proprement dit; il ne peut qu'être excité, éveillé, comme tout ce qui procède de l'esprit ${ }^{17}$, par quelque chose d'extérieur à lui.

Le lecteur assiste donc à une mise en abyme du paysage, qui échappe à la description. Si devant ces paysages toujours grandioses les mots font défaut et si l'écrivain ressent avec force son impossibilité à dire, c'est qu'il ne s'agit pas tant de parler de la nature et des lieux jusque-là inconnus que d'avancer, comme on l'a dit, dans une sorte de quête de soi qui trouve son expression dans l'énorme et l'indéterminé. Face à ces sites l'homme découvre sa petitesse, son néant.

Avant de nous arrêter sur «l'énorme», remarquons le peu de réalité de ces paysages, et ce non seulement à cause d'une rhétorique de l'ineffable, de l'hyperbole, de l'indéterminé, mais aussi parce qu'ils sont situés hors de la réalité disons diurne: ils appartiennent à un monde halluciné. D’où le nombre de syntagmes ou de phrases du type: «cette illusion s'empara de moi» $(L V, 673)$, «un instant j'ai oublié où j'allais [...], j'allais comme dans un rêve» $(L V, 879-880)$, «nous semblions flotter nousmêmes comme des nuages» (HM, 1176). En s'éloignant de la représentation, donc de la mimésis, ces descriptions viseraient non pas la réalité observée, mais la réalité recherchée par l'esprit du narrateur, montrant ainsi sur le coup «l'évanouissement progressif de la réalité objective vers la rêverie subjective» ${ }^{18}$, seul domaine, celui de la subjectivité, de l'irrationnel, d'où l'âme puisse accéder à l'Ábsolu.

Comme tout caractère religieux, dans ses récits autobiographiques George Sand semble vouloir naître à un monde autre, à une nouvelle existence où l'espace n'est pas homogène, mais présente au contraire des ruptures, des cassures, avec des espaces forts, significatifs, c'est-à-dire, sacrés, et des espaces non-consacrés, sans

(15) «Au reste on sait déjà ce que j'entends par un beau pays. Jamais pays de plaine, quelque beau qu'il fût, ne parut tel à mes yeux. Il me faut des torrents, des rochers, des sapins, des bois noirs, des montagnes, des chemins raboteux à monter et à descendre, des précipices à mes côtés qui fassent bien peur» (Euvres Complètes I - Les Confessions (Livre 4), Paris, Gallimard, 1995, «La Pléiade», p. 172).

(16) N'oublions pourtant pas qu'à cette époque le sublime, jusqu'alors catégorie esthétique, commençait à s'ouvrir sur l'ordre du métaphysique (LE SCANFF, op. cit., p. 158).

(17) R. Отто, op. cit., p. 21.

(18) Y. Le SCANFF, op. cit., p. 140. Voir aussi, de ce même auteur, Les “Lettres d'un voyageur" de George Sand. Une poétique romantique du paysage, «Recherches \& Travaux» 70, 2007, pp. 167-180. 
structure ni consistance, amorphes ${ }^{19}$. Le chemin qui jusque-là était entrave ${ }^{20}$ devient libre d'obstacles lorsqu'il conduit vers ce point fixe ${ }^{21}$ qui oriente l'homme religieux dans l'amorphe: «je traversais ce désert sans rencontrer un seul accident qui dérangeât mon rêve» $(L V, 673)$. Les topoi propres au paysage sublime sont bien présents dans les récits de voyage de George Sand. Or cette «masse de roches tendres», «ces piliers», «ces angles des montagnes» $(L V, 893)$ marquent surtout des lieux de coupure, de révélation du sacré. Aussi fermer les yeux «au pied d'une roche» $(L V, 673)$, et non dans un lieu quelconque, permet à l'esprit de divaguer... Dans ce sens il faut signaler d'emblée l'omniprésence du rocher et de la cime, toujours bien délimités. Le premier noir, granitique, éveille la peur, mais aussi le bien-être, la joie au plus profond de l'âme assoiffée:

En face de la grotte [...] s'élève un géant sublime, un rocher perpendiculaire, taillé par les siècles sous la forme d'une citadelle flanquée de ses tours et de ses bastions [...]. Contempler ce pic terrible, du fond de la grotte, au bord de la source, les pieds sur un tapis de violettes, entre la fraîcheur souterraine du rocher et l'air chaud du vallon, c'est un bien-être, c'est une joie, que j'aurais voulu me retirer pour te l'envoyer. $(L V, 666-667)$

La cime, au contraire, enneigée, marque les limites entre le monde matériel et un au-delà qui baigne dans les brumes, dans les nuages, dans l'horizon éthéré: «La chaîne du Mont-Blanc montrait une de ces dernières ceintures granitiques, noire comme l'encre et couronnée de neige» $(L V, 902)$. La verticalité du «rocher perpendiculaire» ou du «pic terrible», aussi bien que l'horizontalité de la chaîne du Mont Blanc viennent délimiter un espace vrai, éloigné en tout de cet autre espace informe, non-homogène, d'ici-bas. Écrit pendant le séjour de Sand à Majorque et intimement lié à l'expérience de ce voyage, Spiridion est lui-même riche d'images qui viennent souligner l'existence de ces deux espaces: «Alors je me levai et me mis à marcher sur le bord du rocher, si rapidement et si près de l'abîme, que j'ignore comment je n'y tombai pas [...]. Je reconnus alors l'Esprit et, saisi de crainte, je m'arrêtai en disant $[\ldots] \gg^{22}$.

Il s'agit bien de sites privilégiés devant lesquels l'homme peut faire l'expérience du sacré, de cet infini divisé par Kant ${ }^{23}$ en un infini mathématique et un infini dynamique. Le premier aurait à voir avec l'expérience que l'homme fait de sa petitesse devant ces grandes montagnes escarpées ou tout ce qui, dans la nature, est immense; le second est lié à tout ce qui, dans la nature, met les hommes face à leur faiblesse: l'orage, le volcan ou tout ce qui relève des forces naturelles qui suscitent l'effroi, en général celles présentes déjà dans les Saintes Écritures ${ }^{24}$. C'est bien ce que Rudolf

(19) M. Eliade, SP, p. 21.

(20) «Quand nous y sommes revenus le soir, plus de champs, plus de chemins» $(C, 552)$.

(21) Voir M. Eliade, op. cit., pp. 21-25. Pour l'homme religieux, continue-t-il, «cette non-homogénéité spatiale se traduit par l'expérience d'une opposition entre l'espace sacré, le seul qui soit réel, qui existe réellement, et tout le reste, l'étendue informe qui l'entoure».

(22) G. SAnd, Spiridion, Paris, Michel Lévy frères, 1867, p. 391. Voyons un deuxième exemple: «[...] et je pouvais admirer à loisir [...] le spectacle sublime de la mer brisant sur les rochers [...]. Là, perdu dans des rêveries sans fin, il me semblait saisir des harmonies inappréciables [...]». Ibidem, p. 367 . Nous faisons ici une exception au dessein de ne nous référer qu'aux œuvres autobiographiques de l'auteur, surtout à celles ayant rapport au voyage. Mais ce livre étrange, Spiridion, le permet, tellement il garde des affinités avec Un biver à Majorque. Â ce propos voir A. SANTA, La estancia en Mallorca de George Sand y "Spiridion", dans C. Vicens-Pujol, George Sand, 1804-2004. L'île et la dame de Nohant, Barcelone, PPU, 2004.

(23) Dans Critique de la faculté de juger (1790). Cité par WunEnburger, op. cit., p. 16.

(24) Voyons un exemple: «Il dit: "Sors. Tiens-toi sur la montagne, face à Yahvé”. Et voici Yahvé passe. 
Otto a appelé le sentiment de l'état de créature, entendu comme «le sentiment de la créature qui s'abîme dans son propre néant et disparaît devant ce qui est au-dessus de toute créature ${ }^{25}$. Cette expérience est maintes fois reprise dans les récits viatiques de notre auteur, comme dans cette première lettre d'un voyageur citée ci-dessus: «Le froid me gagna peu à peu. Il me sembla que, moi aussi, je me pétrifiais. Il me revint à la mémoire je ne sais quel fragment d'un livre inédit: «[...] Il te tarde de tomber en poussière $[\ldots]$. Reste, reste dans ton néant $[\ldots] \gg .(L V, 667)$

L'impression d'infini augmente chez le voyageur dès que la mer devient ellemême objet de contemplation. Ce qui était énorme et pourtant limité, devient alors illimité, aucune frontière ne sépare la terre du ciel ou de la mer, tout devient indifférencié:

Des arbres d'une vigueur prodigieuse mais tout déjetés et à moitié déracinés par les vents, se penchaient sur l'abîme et du fond de cet abîme une autre montagne s'élevait à pic jusqu'au ciel, une montagne de cristal, de diamant et de saphir. La mer, vue d'une hauteur considérable, produit cette illusion, comme chacun sait, de paraitre un plan vertical. $(H M, 1170)$

Dans ce cas-ci horizontalité et verticalité ont cessé d'être le «cadre» de ce qui est vu, même du vécu, pour participer de cette indifférenciation fondamentale qui caractérise l'Absolu. Les plaines de l'Auvergne, quant à elles, s'apparentent à la mer tandis qu'à Majorque la mer et la plaine, on l'a vu, se confondent ${ }^{26}$; la plaine se revêt ainsi des particularités de l'élément liquide et par là même la mer, le lac, ont quelque chose de terrestre. Tout devient ainsi plus illimité:

Ce fond lumineux c'est la plaine; et à cette distance, lorsque les vapeurs de la montagne commencent à s'exhaler et à jeter un voile transparent sur l'abîme, on croirait que c'est déjà la mer. Mais la mer est encore plus loin et, au retour du soleil, quand la plaine est comme un lac bleu, la Méditerranée trace une bande d'argent vif aux confins de cette perspective éblouissante. $(H M, 1117)$

Lors de sa quête, le narrateur tourne ainsi en rond autour de sites toujours identiques $^{27}$ et qui confondent les sens. L'uniformité, la monotonie même de ces lieux où le terrestre se continue dans le maritime, le maritime dans le terrestre, n'est qu'une façon autre de représenter l'infini. En outre le narrateur fait souvent appel à l'aspect sauvage et monstrueux des lieux. Ainsi une tempête qui se déchaîne au pied des Alpes, où le Rhône «précipite son onde impétueuse» et où les arbres sont arrachés «avant d'avoir acquis la force pour résister au choc» $(L V, 851-852)$ ne semble guère éloignée d'une autre au cœur de la Méditerranée: «Alors la montagne ruisselante de cascades et les arbres déracinés par la tempête nous donnaient l'idée du chaos». (HM, 1175)

C'est bien à la manifestation de l'irae Deus, toujours identique à elle-même, que renvoient ces passages, surtout si l'on ajoute des impressions sonores, comme le

Un souffle, grand et fort, ébranle les montagnes, brise les rochers, face à Yahvé. Pas dans le souffle, Yahvé. Et après le souffle, un séisme. Pas dans le séisme, Yahvé. Après le séisme, un feu. Pas dans le feu, Yahvé. Après le feu une voix, un silence subtil» (I Rois 19,11-13). Nous soulignons.

(25) R. Otто, op. cit., p. 24. Et il ajoute: «Le sentiment de l'état de créature [...] est pour ainsi dire l'ombre d'un autre sentiment, celui de "l'effroi", qui, sans aucun doute, se rapporte d'emblée et directement à un objet existant en dehors du moi. Cet objet c'est précisément l'objet numineux». Ibidem, p. 25.

(26) Voir par exemple $V A, 512$ ou HM, 1166.

(27) Il n'en reste pas moins que le narrateur souligne parfois des différences entre les sites. Ainsi le silence de Venise est différent de celui de Barcelone, de Genève, de Majorque (HM, 1065) et les paysages de l'île lui semblent beaucoup plus libres et sauvages que, par exemple, ceux de la Suisse (HM, 1113). 
mugissement du torrent ou la fureur du vent devant lequel «l'âme se resserre» et le voyageur éprouve «un sentiment d'effroi insurmontable [qui] vient glacer le cœur» et lui paraît «horrible et délicieux en même temps» $(H V, 60)$.

\section{Paysage et hiérophanie}

Comme s'il s'agissait de n'oublier aucun des lieux communs des paysages sublimes, aucun des poncifs signalés par Burke, cette peur exprimée au moyen de l'oxymore est également présente devant l'excès de silence ou de solitude ${ }^{28}$, une solitude et un silence, mais aussi une obscurité, qui semblent faits pour mieux prier Dieu, puisqu'il semble bien que c'est de Dieu, ou du divin, qu'il s'agit. «Quelles solitudes austères j'y ai trouvées [au Tyrol] pour prier Dieu!» $(L V, 658)$, s'exclame le narrateur dans la première des Lettres d'un voyageur.

Bien présent dans l'œuvre autobiographique de George Sand, le mot Dieu est normalement associé à des espaces solitaires où la tempête se déchaîne, vient obscurcir le jour et éveiller la crainte dans l'âme du voyageur. C'est le caractère tremendum du sentiment religieux (inséparable pourtant du fascinans), à la fois séduisant et étrangement ravissant ${ }^{29}$. Cet aspect rassurant invite l'homme à continuer sa quête. Et bientôt un glissement s'opère dans le texte, allant de la description d'un paysage sublime à la description de l'âme de celui qui contemple: les mots qui servaient à dire le paysage servent maintenant à dire l'âme mais aussi, et surtout, l'expérience du sacré.

L'expérience religieuse est néanmoins indicible; elle ne peut s'exprimer que par le silence. Celui de Dieu, d'un côté, qui se dérobe à toute saisie: «Si tu me parlais, ô mon Dieu [...]. Hélas, si tu me disais seulement cela» $(S H, 604)$, «Ô Dieu, qui m'as abandonné» $(L V, 778)$, sont des mots très proches de ceux du Christ au moment de sa mort. Or, comme le narrateur semble bien l'avoir compris, ce silence porte en lui la réponse, la seule réponse: «ô silence effrayant, réponse éloquente et terrible de l'éternité!» $(L V, 795)$. Nous pourrions nous référer également au silence qui sert à nommer Dieu, voire à empêcher de le nommer, ce qui a été bien ressenti par les poètes mystiques ${ }^{30}$.

En tout cas cette théologie de la négation propre aux mystiques (le mysticisme étant caractérisé, selon Rudolf Otto, par la prédominance d'éléments irrationnels dans l'approche de Dieu) trouve un parallélisme évident avec les textes sandiens par le biais de l'irreprésentable. Dans ce sens signalons que le motif du nuage - et avec lui, celui de l'orage et du voile -, très présent dans la symbolique chrétienne, tiendra un rôle important dans la construction de ces paysages chargés de sacralité:

(28) «J'admire cette horreur du silence et de la solitude qui pénètre l'âme de terreur et l'absorbe de rêverie» $(V A, 512)$; «Pourquoi cet amour et en même temps cette terreur que nous inspire la solitude?» $(L V, 822)$

(29) R. Отто, op. cit., p. 58.

(30) Si l'étude de l'écriture mystique élargirait trop le cadre de cet article, nous tenons à laisser quelques exemples. Sur le caractère innommable de Dieu, Angelus Silesius (1624-1677) nous laissa un exemple précieux: «Homme, si tu veux exprimer l'essence de l'éternité I il te faut d'abord renoncer au langage» (El peregrino querúbico, Madrid, Siruela, 2005). Dans d'autres cas c'est moyennant l'oxymore que Dieu est désigné, comme le montrent bien ces exemples, de Maître Ekhart (1260-1328): «C'est loin, c'est près | c'est profond et c'est haut I c'est lumière | c'est clarté I c'est ténèbres» (El fruto de la nada, Madrid, Siruela, 2011, p. 141); à Saint Jean de la Croix (1542-1591): «Ô cautère suave l ô délicieuse plaie l tuant, la mort en vie tu as changé» (Poesía completa, Barcelona, Planeta, 1996, p. 339). Nous traduisons. 
Je cherchais Dieu dans le rayon d'une étoile et je me souviens que dans les sombres nuits de l'automne je voyais des monceaux de nuages lourds courir sur ma tête et me voiler le firmament. Hélas, me disais-je, c'est ainsi que tu m'échappais toujours, ô toi que je poursuis! $(\mathrm{SH}, 603)$

Comme chacun sait, la présence de Dieu caché derrière un nuage est une image récurrente dans l'Ancien et le Nouveau Testament: «Moïse monte sur la montagne. Et la nuée couvre la montagne [...]. La nuée la recouvrira six jours. Et il appelle Moïse le septième jour, du milieu de la nuée» (Ex 24, 15-16), lit-on dans l'Exode. Il s'agit là d'une représentation de la transfiguration qui nous semble bien trouver une correspondance dans les textes sandiens. En effet, une lecture intertextuelle permettrait de voir, outre les amples connaissances que l'auteur avait des Saintes Écritures, combien les textes sandiens font appel aux images de la théophanie. La scène revient plusieurs fois: lors d'une de ses promenades, l'âme du voyageur sombre dans l'abîme, la tempête couvre le ciel et une voix se fait entendre tout comme celle, imposante, de la Bible. Voyons côte à côte quelques exemples tirés des textes de Sand et des Saintes Écritures:

Une grande voix s'éleva dans l'orage et je l'entendis par-dessus toutes les autres: «Allons, mes âmes, disait cette voix terrible, combattez courageusement» $(S H, 612)$.

Tu lutteras en vain contre la grande voix de l'univers [...] et l'homme [...] sera toujours saisi d'épouvante quand il voudra interroger ce qui est au-dessus de lui. $(L V, 795)$

Et une nuée survint qui les prit sous son ombre, et une voix partit de la nuée: «Celui-ci est mon Fils bien-aimé; écoutez-le». $(M c, 9,7)$

La foule, qui se trouvait là et qui avait entendu, disait qu'il y avait eu un coup de tonnerre. $(\mathrm{J} n, 12,29)$

ce tonnerre symbolisant bien la voix de Dieu: «Moïse parlait, et Dieu lui répondait par des coups de tonnerre» $(E x, 19,19)$.

Comme l'orage, le nuage est à la fois obscurité et lumière: présence invisible (obscurité) de Dieu, dont on entend (lumière) la voix. L'un et l'autre sont bien des signes théophaniques qui montrent et cachent à la fois la présence de Dieu, porteur d'un message divin. Cette contradiction fondamentale présente dès les Saintes Écritures deviendra par la suite récurrente chez les écrivains mystiques ${ }^{31}$.

Les nuages sombres, les tempêtes si chères aux romantiques et, semble-t-il, nécessaires pour sonder les abîmes de l'esprit, terribles parce qu'inconnus, deviennent chez George Sand des métaphores de ce dieu tantôt caché, tantôt voilé, tantôt illuminé d'une lumière blême mais jamais tout à fait visible. Tout comme le rocher ou la cime le nuage (mais aussi le voile) vient marquer une limite entre la réalité d'icibas et le sacré. Ces paysages, toujours étranges et merveilleux, deviennent ainsi une véritable hiérophanie: à travers l'orage, image également du chaos primitif (et par là même rattachée à la Genèse), c'est la voix divine qui se fait présente, qui invite ceux qui cherchent à combattre l'ouragan, car «ma tempête est rude» $(S H, 612)$. Notons aussi que dans la première Lettre le «gémissement du vent dans le feuillage lourd des oliviers» $(L V, 660)$ transporte le narrateur jusqu'au Jardin des Oliviers ${ }^{32}$ où le Christ

(31) Permettons-nous deux exemples, Thérèse d'Ávila parlera d'«Une nuée d'une immense clarté», op. cit., p. 197 et Saint Jean de la Croix écrira, à son tour: «Plus on monte haut I moins on comprend I que c'était la nuée ténébreuse | qui éclaircissait la nuit», op. cit., p. 18.

(32) Cet épisode est par ailleurs cher aux poètes romantiques. Rappelons par exemple «Le Crucifix», de Lamartine; «Le Christ aux Oliviers» de Nerval ou encore «Le Mont des Oliviers» de Vigny. 
pria toute une nuit, alors que le destinataire de la lettre lutte contre ce «géant aux ailes de feu» sans pouvoir «soutenir l'éclat de sa face» $(L V, 661)$. Il s'agit bien de Jacob qui, lui aussi, lutta contre l'ange au milieu d'une nature sauvage, voire hostile: une des premières phrases de Jacob n'est-elle pas: «Que ce lieu est redoutable!»? $(G n, 28,17)$.

Si l'imaginaire chrétien trouve une place importante dans l'univers sandien c'est bien une rencontre avec le sacré, plus qu'avec une religion quelconque $e^{33}$, qui nous est proposée. En ceci George Sand s'éloigne d'autres écrivains romantiques qui ont également fait l'expérience de la sublimité mais chez qui l'infini «devient vacuité», «est ressenti comme le vide, le vidé» ${ }^{34}$. Ainsi, après une analyse de l'expression du sublime dans l'œuvre de Victor Hugo, Yvon Le Scanff conclut que

le sublime n'est donc pas au service de la religion qui méconnaît sciemment l'infini, il s'apparente davantage à une expérience esthétique et métaphysique où l'intuition du sacré évite au sublime de se dégrader en un simple jeu esthétique, où le sublime reste le garant du droit de l'homme à une jouissance surhumaine, à la contemplation libre du visible infini, mystérieux et finalement indéterminé $e^{35}$.

L'infini sandien nous semble être, au contraire, au service sinon de la religion, du moins du sacré. Le sentiment de l'infini, on l'a dit, se trouve non pas dans les paysages contemplés mais dans l'âme de celui qui contemple, de telle sorte que la déchirure qui unit l'homme au sacré est (ou n'est pas) consubstantielle à l'homme: «Vous savez bien, malheureux hommes, qu'il y a un orage sur votre tête, et qu'il éclatera inévitablement» $(S H, 595)$. Dès ce jour- là, conclut George Sand, celui qui n'est pas religieux «sera tout à fait perdu» $(\mathrm{SH}, 595)$.

\section{Pour conclure}

Très jeune, et Corambé en est bien la preuve, George Sand a laissé voir une spiritualité qui s'accommodait mal des exigences de solitude et de refus du monde matériel et du moi qui sont celles du christianisme. Elle rêve plutôt d'une nouvelle Église, détaillée dans Le Meunier d'Angibault, Lélia, Spiridion, Lettres à Marcie et Consuelo ${ }^{36}$. De ces pages se dégage une idée de la figure du Christ et d'un certain catholicisme à inflexion socialiste qui accompagnera l'écrivain tout au long de sa vie. Or il s'agit là d'une approche rationnelle du sacré, de l'idée de Dieu.

$\mathrm{Au}$ contraire, nous avons voulu nous pencher dans les pages précédentes sur l'aspect irrationnel de l'expérience religieuse de George Sand, qu'il nous a été possible d'entrevoir à travers les descriptions de paysages aussi bien dans ses récits de

(33) «L'expérience du sacré se distingue donc de l'approche théologique de Dieu, dans la mesure où elle ne fait que préparer la place d'une altérité, qu'annoncer la présence d'une plénitude, mais qui restent toutes deux encore indéterminées». J.-J. WunENBURGER, op. cit., p. 15.

(34) Y. LE SCANFF, op. cit., p. 134. En effet nous préférons pour parler du sacré chez Victor Hugo le mot panthéisme. En ceci la lettre XX de Le Rhin nous semble assez illustrative: «Vous savez qu'il y a des moments où je crois presque à l'intelligence des choses; il me semblait qu'une foule de voix murmuraient dans ce ravin et me disaient: - Où vas-tu? tu cherches les endroits où il y a peu de pas humains et où il y a beaucoup de traces divines [...]; tu cherches le lieu [...] où l'on sent l'éternité au fond [...]; tu cherches de l'herbes et des mousses, des feuilles humides, des branches gonflées de sève, des oiseaux qui fredonnent [...]» (Hetzel, 1842, vol 2, p. 18).

(35) Ibidem, p. 184.

(36) M.F. BOWMAN, op. cit., p. 247. 
voyage, genre à composante hautement autobiographique que, dans une moindre mesure, dans l'ensemble de son œuvre autobiographique.

Si dans un premier temps le lecteur est bien devant le paysage du sublime romantique, à la fois terrible et attirant, énorme dans le sens de «tout ce qui n'est pas normal, ce qui est troublant $\gg^{37}$ et qui met l'homme face à sa petitesse, il se rend compte rapidement que la description des paysages dépasse la simple esthétique du sublime sombre pour atteindre une dimension plus spirituelle, celle de l'écriture mystique et de l'expérience du numineux. Dans ce sens, nous avons voulu mettre l'accent sur ce qui, au sein de l'amorphe, de l'indéterminé, guide le voyageur dans sa quête, telles ces «saillies anguleuses des rochers» $(S H, 613)$ qui séparent avec netteté l'espace nonconsacré (donc profane) de l'espace sacré. À souligner encore que le rocher joue un rôle central dans la symbolique du Christ depuis que Moïse le frappa de son bâton. N'est-ce pas à cette image que l'écrivain fait appel lorsqu'elle écrit: «[...] avec le pouvoir de faire sortir des larmes de charité des entrailles du roc» (LV, 819)? Paradoxalement la valorisation de l'indéterminé semble bien traduire un élan vers l'infini, vers ce Tout-Autre qui ne connaît pas de bornes.

Une forte présence de la symbolique chrétienne - tel le nuage ou le rocher -, ainsi que des thèmes et des traits stylistiques propres à l'écriture mystique - la rhétorique de la négation, de l'oxymore -, semblent corroborer qu'on est passé du sublime au numineux, ce qui ne peut étonner si on tient compte du fait que «le sentiment du sublime a certainement été l'un de ces facteurs excitatifs qui ont servi à éveiller le sentiment du numineux» ${ }^{38}$. Le sublime déborderait ainsi sa fonction esthétique pour introduire au sacré, et ferait en même temps le lien entre les deux niveaux: formel (esthétique) et sacré (contenu). 